\title{
Estimates of weak and electromagnetic nuclear decay signatures for neutrino reactions in Super-Kamiokande
}

\author{
E. Kolbe \\ Physics Division, Oak Ridge National Laboratory, Oak Ridge, Tennessee 37831-6373 \\ K. Langanke \\ Institut for Fysik og Astronomi, Århus Universitet, DK-8000 Århus C, Denmark \\ P. Vogel \\ Department of Physics, California Institute of Technology, Pasadena, California 91125 \\ (Received 2 October 2001; revised manuscript received 26 April 2002; published 24 July 2002)
}

\begin{abstract}
We estimate possible delayed $\beta$ decay signatures of the neutrino induced reactions on ${ }^{16} \mathrm{O}$ in a two-step model: the primary neutrino $(\nu, l)$ process, where $l$ is the lepton in the final state, is described within the random phase approximation, while the subsequent decay of the excited nuclear state in the final channel is treated within the statistical model. We calculate partial reaction cross sections leading to $\beta$ unstable nuclei. We consider neutrino energies up to $500 \mathrm{MeV}$, relevant for atmospheric neutrino detection in Super-Kamiokande, and supernova neutrino spectra.
\end{abstract}

DOI: 10.1103/PhysRevD.66.013007

PACS number(s): 13.15.+g, 14.60.Pq, 25.30.Pt, 96.40.Tv

The observation of neutrino-induced events by the SuperKamiokande (SK) Collaboration has contributed decisively to the understanding of several fundamental physics questions: e.g., the atmospheric neutrino puzzle, related to neutrino oscillations [1], the confirmation of the deficit of solar neutrinos compared to expectations from solar models [2], and the first detection of supernova neutrinos by its smaller predecessor [3] which has supported the basic understanding of core collapse supernovae. The main neutrino detection scheme in SK are $\nu$-electron elastic scattering, $\nu$ absorption on free nucleons, and $\nu$ quasielastic charged-current scattering on ${ }^{16} \mathrm{O}$.

However, other neutrino induced reactions are of interest as well. For example, in Ref. [4] it has been shown that $\gamma$ rays produced by inelastic $\nu$ scattering off ${ }^{16} \mathrm{O}$, followed by nucleon emission through particle-bound excited states in ${ }^{15} \mathrm{O}$ and ${ }^{15} \mathrm{~N}$, can be used as signal for supernova $\nu_{\mu}$ and $\nu_{\tau}$ neutrinos and antineutrinos in SK. Clearly, many of the primary neutral- and charged-current $\nu$-induced reactions in SK occur on ${ }^{16} \mathrm{O}$. Atmospheric neutrinos have high enough energies so that the final nucleus in the primary reaction will be in an excited state which will then decay by a cascade of particle and $\gamma$ emissions. Nussinov and Shrock [5] stressed that these cascades can result in the production of $\beta$-unstable nuclei. If the $Q_{\beta}$ values of these nuclei are above the observational threshold energy in $\mathrm{SK}(\sim 5 \mathrm{MeV})$, these decays might be detectable, and since they are usually delayed, might offer an additional characteristic signature of the neutrino-induced reactions. As possible candidates ${ }^{16} \mathrm{~N}$ $\left(T_{1 / 2}=7.13 \mathrm{~s}, Q_{\beta}=10.4 \mathrm{MeV}\right),{ }^{15} \mathrm{C}\left(T_{1 / 2}=2.4 \mathrm{~s}, Q_{\beta}=9.8\right.$ $\mathrm{MeV}),{ }^{12} \mathrm{~B}\left(T_{1 / 2}=20.2 \mathrm{~ms}, Q_{\beta}=13.4 \mathrm{MeV}\right)$, and ${ }^{12} \mathrm{~N}\left(T_{1 / 2}\right.$ $=11 \mathrm{~ms}, Q_{E C}=17.3 \mathrm{MeV}$ ) have been identified. Nussinov and Shrock have estimated the expected event rates for reactions in SK induced by atmospheric neutrinos leading to such nuclei on the basis of continuum random phase approximation (CRPA) calculations performed for Liquid Scintillation
Neutrino Detector (LSND) neutrino beams and a ${ }^{12} \mathrm{C}$ target [6]. Reference [5] called for improved calculations of the nuclear physics pathways to the various unstable nuclei. We have performed such calculations, adopting the same approach as already used in Ref. [4].

Reference [5] also mentions that the same signature could serve as an additional signal for supernova neutrino detection in Super-Kamiokande. We have therefore also calculated the relevant partial reaction cross sections for neutrino energies expected in the core collapse supernova bursts. Typically, supernova models predict a neutrino energy hierarchy in which the average neutrino energies follow the $\bar{E}_{\nu_{e}}<\bar{E}_{\nu_{e}}$ $<\bar{E}_{\nu_{x}}$ pattern, where $\nu_{x}$ is a shorthand for $\nu_{\mu}, \nu_{\tau}$ and their antiparticles which are expected to have identical spectra. This hierarchy, however, could be modified by neutrino oscillations. Without oscillations, due to the rather high particle threshold in ${ }^{16} \mathrm{O}$, only supernova $\nu_{x}$ will react appreciably with ${ }^{16} \mathrm{O}$ in the water Cerenkov detectors by the neutral current interactions. However, if $\nu_{e} \leftrightarrow \nu_{\mu}$ and/or $\bar{\nu}_{e} \leftrightarrow \bar{\nu}_{\mu}$ oscillations occur, the $\nu_{e}$ and $\bar{\nu}_{e}$ neutrinos can induce chargedcurrent reactions due to their increased energy. We have studied this possibility as well.

To calculate the various partial neutrino-induced reaction cross sections for neutrino-induced reactions on ${ }^{16} \mathrm{O}$ we assume a two-step process. In the first step we calculate the charged current $(\mathrm{CC}){ }^{16} \mathrm{O}\left(\nu_{l}, l^{-}\right){ }^{16} \mathrm{~F}^{*}$ and ${ }^{16} \mathrm{O}\left(\bar{\nu}_{l}, l^{+}\right){ }^{16} \mathrm{~N}^{*}$ cross sections (where $l=e$ or $\mu$ ), and the neutral current (NC) cross section ${ }^{16} \mathrm{O}\left(\nu, \nu^{\prime}\right){ }^{16} \mathrm{O}^{*}$, as a function of excitation energy in the final nucleus. These calculations have been performed within the CRPA using the finite range force based on the Bonn potential [7] and considering all multipole operators with $J \leqslant 9$ and both parities. The CRPA model is described in Ref. [8]. In the second step we calculate for each final state with well-defined energy the branching ratios into the various decay channels using the statistical model code SMOKER [9]. As possible final states in the residual nucleus the SMOKER code considers the experimentally 


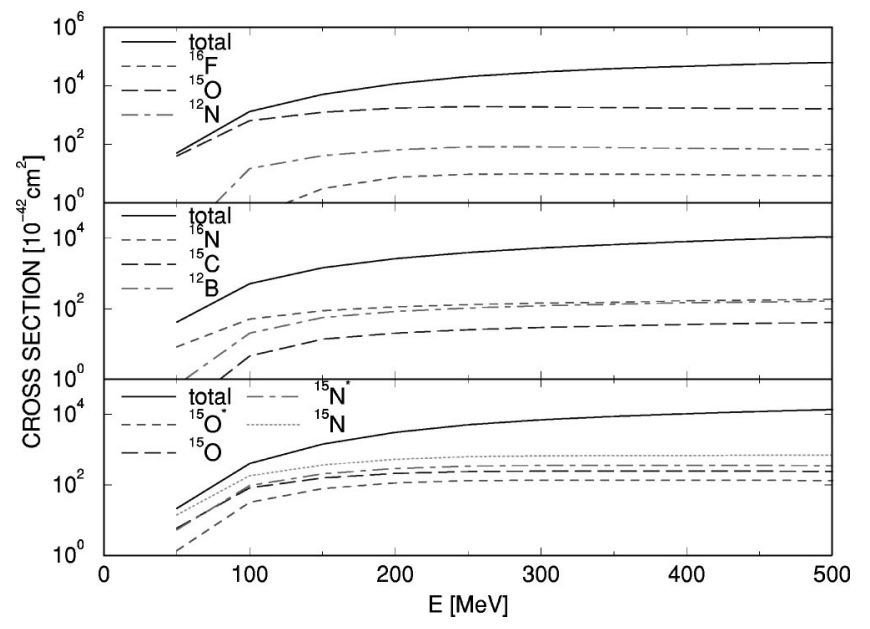

FIG. 1. Total and partial cross sections to selected $\beta$-unstable nuclear ground states for $\left(\nu_{e}, e^{-}\right)$(upper), $\left(\bar{\nu}_{e}, e^{+}\right)$(middle), and $\left(\nu, \nu^{\prime}\right)$ (lower part) reactions on ${ }^{16} \mathrm{O}$ as function of neutrino energy. As explained in the text, the symbols ${ }^{15} \mathrm{O}^{*}$ and ${ }^{15} \mathrm{~N}^{*}$ in the lower panel denote the partial cross sections leading through particlebound excited states in these nuclei.

known levels supplemented at higher energies by an appropriate level density formula [9]. As decay channels proton, neutron, $\alpha$ and $\gamma$ emission are included in the code. If the decay leads to an excited level of the residual nucleus, we calculate the branching ratios for the decay of this state in an analogous fashion [4]. Using the appropriate branching ratios and the corresponding primary charged- or neutral-current cross sections, we determine the various partial particle emission cross sections.

We note that our model has been previously used to calculate various neutrino-induced reaction cross sections and muon capture rates. For the present application it is important that the model describes both the total muon capture rate on ${ }^{16} \mathrm{O}$ as well as the partial captures to particle-bound states in ${ }^{16} \mathrm{~N}$ quite well $[10,11] .{ }^{1}$

Figure 1 shows the partial $\left(\bar{\nu}_{e}, e^{+}\right)$and $\left(\nu_{e}, e^{-}\right)$cross sections leading to the $\beta$-unstable ${ }^{16} \mathrm{~N},{ }^{15} \mathrm{C}$, and ${ }^{12} \mathrm{~B}$ and ${ }^{16} \mathrm{~F}$ (which promptly decays to $p+{ }^{15} \mathrm{O}$ ), ${ }^{15} \mathrm{O}$, and ${ }^{12} \mathrm{~N}$ ground states in the final channel; these cross sections reflect the sum over all cross sections with particle-bound states of these nuclei as these excited states will fast decay to the ground state by $\gamma$ emission. Our calculations have been performed up to neutrino energies $E_{\nu}=500 \mathrm{MeV}$. At higher energies the total cross sections can be obtained from a relativistic Fermi gas model [12], including, however, additional channels such as pion production.

The results allow us to draw some interesting conclusions. While the total cross sections increase with neutrino energies, most of this increase goes into new channels which open up with increasing neutrino energy and the partial cross sections leading to definite states have the tendency to saturate. Thus our partial cross sections to these states, obtained

\footnotetext{
${ }^{1}$ The partial rates listed in Table II of Ref. [10] contain a typographical error. They should be multiplied by a factor 1000 .
}

for, say, $E_{\nu}=500 \mathrm{MeV}$, can be used to derive upper limits for the corresponding branching ratios expected for atmospheric neutrinos which, on average, have even larger energies.

The ${ }^{16} \mathrm{O}\left(\bar{\nu}_{e}, e^{+}\right)$reaction leading to particle-bound states in ${ }^{16} \mathrm{~N}$ deserves special attention. In addition to the ${ }^{16} \mathrm{~N}$ ground state (with $J^{\pi}=2^{-}$) there are $0^{-}, 3^{-}$, and $1^{-}$states below the neutron threshold which will decay by $\gamma$ emission to the $\beta$ unstable ${ }^{16} \mathrm{~N}$ ground state. In muon capture the particle-bound states contribute about $10 \%$ of the total rate, with the ground state dominating, in close agreement between data [13] and calculation [10]. The situation is similar in the $\left(\bar{\nu}_{e}, e^{+}\right)$reaction. For $E_{\nu}=100 \mathrm{MeV}$, which resembles the energy transfer encountered in muon capture, we find that about $10 \%$ of the total cross section leads to particle-bound states in ${ }^{16} \mathrm{~N}$, and hence are followed by a $\beta$ decay of the ${ }^{16} \mathrm{~N}$ ground state. For smaller (higher) neutrino energies, the relative weight of the particle-bound states in phase space increases (decreases). This is also clearly born out in our calculation. For $E_{\nu}=50 \mathrm{MeV}$ nearly $20 \%$ of the total cross section ends finally by $\beta$ decay of ${ }^{16} \mathrm{~N}$. At higher neutrino energies, relevant for atmospheric neutrinos, this percentage drops to about $1.6 \%$ for $500 \mathrm{MeV}$ neutrinos and will be even smaller for more energetic neutrinos. As discussed above, reaction cross section to specific states have the tendency to saturate with increasing neutrino energy. For the partial ${ }^{16} \mathrm{O}\left(\bar{\nu}_{e}, e^{+}\right){ }^{16} \mathrm{~N}$ cross section our calculation yields $\sim(1.4-1.8) \times 10^{-40} \mathrm{~cm}^{2}$ for $E_{\bar{\nu}}=300-500 \mathrm{MeV}$ (see Fig. 1).

The total ${ }^{16} \mathrm{O}\left(\nu_{e}, e^{-}\right)$and ${ }^{16} \mathrm{O}\left(\bar{\nu}_{e}, e^{+}\right)$cross sections for neutrino energies up to $200 \mathrm{MeV}$ have been calculated by Haxton [14] using the nuclear shell model. As noted by the author, the considered allowed and first forbidden response (and model space) is probably sufficient to reliably describe the inclusive neutrino reactions for $E_{\nu} \leqslant 100 \mathrm{MeV}$, while at higher neutrino energies additional nuclear modes (and a larger model space) are required. This is confirmed by our calculations. For $E_{\nu} \leqslant 100 \mathrm{MeV}$ our results agree quite well with those calculated by Haxton [14], while for $E_{\nu}$ $=200 \mathrm{MeV}$ our cross sections are larger than those in Ref. [14] by more than a factor of 2. Haxton also studied the ${ }^{16} \mathrm{O}\left(\bar{\nu}_{e}, e^{+}\right)$reaction leading to the particle-bound states in ${ }^{16} \mathrm{~N}$. It is important to note that this calculation is independent of his restriction in nuclear response and model space. Indeed, Haxton finds an energy dependence of the partial cross section which is rather close to ours, also showing the saturation with increasing energy $E_{\nu}$. Our calculation of the absolute ${ }^{16} \mathrm{O}\left(\bar{\nu}_{e}, e^{+}\right){ }^{16} \mathrm{~N}$ cross section, which has been cross checked against the relevant partial muon capture rates, gives somewhat larger result (by about a factor of 2) than those in Ref. [14].

Nussinov and Shrock [5] assumed that of order $10 \%$ of the $\bar{\nu}_{e}$ induced reactions from the atmospheric neutrino flux will be followed by a $\beta$ decay of ${ }^{16} \mathrm{~N}$ and hence might be a viable additional signal for these reactions in the SuperKamiokande detector. While our calculation confirms the general idea of these authors, it indicates that the relative 
weight of this decay channel is noticeably smaller. Our RPA study suggests that less than about $1.5 \%$ of the reactions induced by antineutrinos with $E_{\bar{\nu}}>500 \mathrm{MeV}$ will lead to the production of ${ }^{16} \mathrm{~N}$ in the final channel. If we adopt this percentage, the estimate of events of this type, as given in Ref. [5], reduces to about 15 or less in the current sample of atmospheric neutrino data in Super-Kamiokande. (This estimate does not include the possible production of ${ }^{16} \mathrm{~N}$ on other ${ }^{16} \mathrm{O}$ nuclei induced by the cascade of nucleons and pions ejected in the primary reaction. Such secondary production was not considered in Ref. [5] either.)

Reactions leading to the ${ }^{15} \mathrm{C}$ ground state are slightly less likely $\left(<0.4 \%\right.$ for $\left.E_{\bar{\nu}}^{-}=500 \mathrm{MeV}\right)$. Another interesting final state is ${ }^{12} \mathrm{~B}$, produced by $\left(\bar{\nu}_{e}, e^{+} \alpha\right)$. The respective partial cross section is about $1.5 \%$ of the total for $E_{\nu}^{-}=500 \mathrm{MeV}$. This is much higher than for the $\nu_{e}$-induced reaction, where the $\alpha$ decay channel to the ${ }^{12} \mathrm{~N}$ ground state contributes only about $0.1 \%$ to the total cross section for $E_{\nu}=500 \mathrm{MeV}$ due to the larger Coulomb barrier. Our partial cross sections leading to ${ }^{12} \mathrm{~B}$ and ${ }^{12} \mathrm{~N}$ are more than an order smaller than the estimates used in Ref. [5], although some of the discrepancy is removed if one normalizes the branching ratios to similar energies. Our partial cross sections scale the expected number of events for the current SK data to the order $<O(10)$. In the $\nu_{e}$-induced reactions most of the $\alpha$ decays go to excited states of ${ }^{12} \mathrm{~N}$ which then dominantly decay by proton emission leading to ${ }^{11} \mathrm{C}$. Unfortunately the $Q_{\beta}$ value of this nucleus $(1.9 \mathrm{MeV})$ is below the observational limit of SK. The same is true for the reactions leading to ${ }^{15} \mathrm{O}$, which contributes about $2.6 \%$ of the total $\left(\nu_{e}, e^{-}\right)$cross section for $E_{\nu}=500 \mathrm{MeV}$.

Our neutral-current cross sections are, as customary, presented as averages of neutrino and antineutrino cross sections (see Fig. 1). The sum of the $\left(\nu, \nu^{\prime} p\right)$ and $\left(\nu, \nu^{\prime} n\right)$ cross sections through the particle-bound excited states in ${ }^{15} \mathrm{~N}$ and ${ }^{15} \mathrm{O}$ (the supernova neutrino signal from Ref. [4] and denoted by ${ }^{15} \mathrm{~N}^{*}$ and ${ }^{15} \mathrm{O} *$ in Fig. 1) increases from $6.7 \times 10^{-42} \mathrm{~cm}^{2}$ for $E_{\nu}=50 \mathrm{MeV}$ to $481 \times 10^{-42} \mathrm{~cm}^{2}$ for $E_{\nu}=500 \mathrm{MeV}$. Nevertheless the relative weight of these channels decreases with increasing neutrino energy. While it contributes more than $30 \%$ for $E_{\nu}=50 \mathrm{MeV}$, its fraction has decreased to $3.5 \%$ for $E_{\nu}=500 \mathrm{MeV}$. We note that, even at $E_{\nu}=500 \mathrm{MeV}$, a large portion of the cross section leads to the production of ${ }^{15} \mathrm{O}$ (more than $5 \%$ ), whose decay is unfortunately not detectable by SK due to its low $Q_{\beta}$ value.

We have repeated all calculations for $\nu_{\mu}$ and $\bar{\nu}_{\mu}$ neutrinos. We find slightly smaller (by about $5 \%$ ) total cross sections for $\mu$ neutrinos than for electron neutrinos, while the relative branchings are nearly unchanged at atmospheric neutrino energies, i.e., $E_{\nu}>300 \mathrm{MeV}$.

It has been suggested that some of the late-time electrons or positrons observed in the Kamiokande from supernova 1987A have arisen from beta decays of nuclei produced by neutrinos that arrived earlier during the interval of supernova neutrino data [15]. Motivated by this intriguing possibility we have calculated partial cross sections for supernova induced reactions on ${ }^{16} \mathrm{O}$ leading to $\beta$-unstable nuclei.
While most supernova models [16-19] agree that the above mentioned neutrino energy hierarchy, $\bar{E}_{\nu_{e}}<\bar{E}_{\nu_{e}}<\bar{E}_{\nu_{x}}$ is expected, there is a considerable uncertainty in the actual values of these average energies. In many applications it is assumed that the supernova neutrino spectra can be represented by Fermi-Dirac (FD) distributions with temperature $T$ and degeneracy parameter $\alpha$. But again, various models predict different values for these parameters. Since our purpose here is to aid future observations by calculating cross sections, we present our results in Table I for a few relevant channels as a function of the incoming neutrino energy. Once there is a consensus on the expected neutrino flux energy profile, it will be easy to perform the corresponding folding.

Similarly, while there is a consensus that the observation of atmospheric and solar neutrinos (see, in particular, Ref. [20]) can be explained only by neutrino oscillations, the actual values of oscillation parameters remain uncertain. The signal of supernova SN1987A [3,21] has been analyzed by many people as a source of information on oscillations [2224]. The conclusions are, however, contradictory, due no doubt to the low statistical significance of the SN1987A signal. It is thus important to explore all means to enhance and quantify the neutrino signal of the future galactic supernova. The possibility of signals with delayed $\beta$ emission is explored here.

In Table I we show the calculated total cross sections for the CC $\nu_{e}$ and $\bar{\nu}_{e}$ induced reactions and for the NC $\nu_{x}$ induced reaction, as well as the partial cross sections leading to the $\beta$ unstable and potentially observable nuclei ${ }^{16} \mathrm{~N},{ }^{12} \mathrm{~B}$, and ${ }^{12} \mathrm{~N}$. As has already been shown by Haxton [14], the thresholds in ${ }^{16} \mathrm{O}$ are too high for supernova $\nu_{e}$ and $\bar{\nu}_{e}$ neutrinos with average energies of about 12 and $15 \mathrm{MeV}$, respectively, to significantly induce charged-current reactions. However, the average energies of $\nu_{x}$ neutrinos is expected to be large enough $\left(\bar{E}_{\nu_{x}} \sim 25 \mathrm{MeV}\right)$ to excite states in ${ }^{16} \mathrm{O}$ above particle thresholds which then decay by particle emission. As can be seen in Table II, which summarizes the total and various partial neutral-current reaction cross sections for the Fermi-Dirac distribution with $T=8 \mathrm{MeV}$ taken as a representative case for the expected spectrum of the $\nu_{x}$ neutrinos, most of these decays (about 85\%) are by single neutron and proton emissions which dominantly lead directly to the ground states of ${ }^{15} \mathrm{~N}$ and ${ }^{15} \mathrm{O}$. However, in an important second branch the particle emission is to particle-bound states in ${ }^{15} \mathrm{~N}$ and ${ }^{15} \mathrm{O}$; the subsequent $\gamma$ decays should be observable in Super-Kamiokande and can serve as a signal for supernova $\nu_{x}$ neutrinos [4]. Other, less important decay branches lead to ${ }^{11} \mathrm{C}$ or ${ }^{14} \mathrm{C}$. But similar to ${ }^{15} \mathrm{O}$, none of these beta unstable nuclei offers an observable delayed signal for the SK detector. Note that the NC reactions are not affected by neutrino oscillations among "active," i.e., nonsterile neutrinos.

While our main results are shown in Table I, Table III shows the total and partial $\nu_{e}$-induced charged-current reaction cross sections, calculated for low $(T=4 \mathrm{MeV})$ and high $(T=8 \mathrm{MeV})$ temperatures, to stress the steep dependence of yield on temperature (or average energy). The par- 
TABLE I. Total and partial cross sections for the indicated reactions as function of the incoming neutrino energy. The cross sections are given in units of $10^{-42} \mathrm{~cm}^{2}$, exponents are given in parentheses, neutrino energy is in MeV. Star means that the partial cross sections for all particle bound states are added together.

\begin{tabular}{ccccccc}
\hline \hline$E_{\nu}$ & ${ }^{16} \mathrm{O}\left(\nu_{e}, e^{-}\right) X$ & ${ }^{16} \mathrm{O}\left(\bar{\nu}_{e}, e^{+}\right) X$ & ${ }^{16} \mathrm{O}\left(\nu, \nu^{\prime}\right) X$ & ${ }^{16} \mathrm{O}\left(\bar{\nu}_{e}, e^{+}\right){ }^{16} \mathrm{~N}$ & ${ }^{16} \mathrm{O}\left(\bar{\nu}_{e}, e^{+} \alpha\right){ }^{12} \mathrm{~B}^{*}$ & ${ }^{16} \mathrm{O}\left(\nu_{e}, e^{-} \alpha\right){ }^{12} \mathrm{~N}^{*}$ \\
\hline 15.0 & $1.56(-6)$ & $2.53(-2)$ & $5.10(-4)$ & $2.53(-2)$ & $0.00(+0)$ & $0.00(+0)$ \\
17.5 & $8.42(-4)$ & $7.27(-2)$ & $3.92(-3)$ & $7.13(-2)$ & $0.00(+0)$ & $0.00(+0)$ \\
20.0 & $7.26(-3)$ & $1.81(-1)$ & $1.60(-2)$ & $1.58(-1)$ & $0.00(+0)$ & $0.00(+0)$ \\
22.5 & $3.99(-2)$ & $4.21(-1)$ & $6.04(-2)$ & $3.00(-1)$ & $1.10(-8)$ & $0.00(+0)$ \\
25.0 & $1.77(-1)$ & $8.90(-1)$ & $1.75(-1)$ & $5.16(-1)$ & $2.31(-4)$ & $0.00(+0)$ \\
27.5 & $5.23(-1)$ & $1.69(+0)$ & $4.13(-1)$ & $8.24(-1)$ & $2.32(-3)$ & $4.28(-5)$ \\
30.0 & $1.25(+0)$ & $2.94(+0)$ & $8.43(-1)$ & $1.24(+0)$ & $8.66(-3)$ & $1.68(-3)$ \\
32.5 & $2.58(+0)$ & $4.76(+0)$ & $1.54(+0)$ & $1.78(+0)$ & $2.20(-2)$ & $8.12(-3)$ \\
35.0 & $4.76(+0)$ & $7.26(+0)$ & $2.59(+0)$ & $2.46(+0)$ & $4.55(-2)$ & $2.22(-2)$ \\
37.5 & $8.05(+0)$ & $1.06(+1)$ & $4.07(+0)$ & $3.29(+0)$ & $8.33(-2)$ & $4.67(-2)$ \\
40.0 & $1.28(+1)$ & $1.48(+1)$ & $6.09(+0)$ & $4.28(+0)$ & $1.40(-1)$ & $8.49(-2)$ \\
45.0 & $2.76(+1)$ & $2.64(+1)$ & $1.21(+1)$ & $6.71(+0)$ & $3.32(-1)$ & $2.16(-1)$ \\
50.0 & $5.21(+1)$ & $4.29(+1)$ & $2.14(+1)$ & $9.77(+0)$ & $6.73(-1)$ & $4.48(-1)$ \\
55.0 & $8.89(+1)$ & $6.46(+1)$ & $3.46(+1)$ & $1.34(+1)$ & $1.22(+0)$ & $8.14(-1)$ \\
60.0 & $1.41(+2)$ & $9.17(+1)$ & $5.24(+1)$ & $1.75(+1)$ & $2.01(+0)$ & $1.36(+0)$ \\
65.0 & $2.12(+2)$ & $1.25(+2)$ & $7.53(+1)$ & $2.20(+1)$ & $3.10(+0)$ & $2.12(+0)$ \\
70.0 & $3.02(+2)$ & $1.63(+2)$ & $1.04(+2)$ & $2.68(+1)$ & $4.52(+0)$ & $3.15(+0)$ \\
80.0 & $5.52(+2)$ & $2.57(+2)$ & $1.78(+2)$ & $3.79(+1)$ & $8.36(+0)$ & $6.07(+0)$ \\
90.0 & $8.92(+2)$ & $3.77(+2)$ & $2.76(+2)$ & $4.92(+1)$ & $1.35(+1)$ & $1.02(+1)$ \\
100.0 & $1.32(+3)$ & $5.18(+2)$ & $3.99(+2)$ & $6.00(+1)$ & $2.01(+1)$ & $1.49(+1)$ \\
\hline \hline
\end{tabular}

tial cross section ${ }^{16} \mathrm{O}\left(\nu_{e}, e^{-} p \gamma\right){ }^{15} \mathrm{O}^{*}$, i.e., the supernova signal of Ref. [4], is rather large. Thus, for large $\bar{E}_{\nu_{e}}$ both charged-current and neutral-current reactions will lead to such a decay mode. These two events can, however, be distinguished in the SK detector as the charged-current events will be accompanied by the production of an electron in the final state, which can be detected, while in the neutralcurrent reaction there is no electron. An interesting further decay branch is given by the ${ }^{16} \mathrm{O}\left(\nu_{e}, e^{-} \alpha\right){ }^{12} \mathrm{~N}$ reaction (see

TABLE II. Partial cross sections for neutral-current neutrinoinduced reactions on ${ }^{16} \mathrm{O}$. A Fermi-Dirac distribution with $T$ $=8 \mathrm{MeV}$ and zero chemical potential is assumed. The cross section, in units of $10^{-42} \mathrm{~cm}^{2}$, is the average for neutrino and antineutrino reactions, and the exponents are given in parentheses. The asterisks indicate that the cross sections have been summed over all particle-bound states.

\begin{tabular}{ll}
\hline \hline Neutrino reaction & $\sigma, T=8 \mathrm{MeV}$ \\
\hline \hline${ }^{\text {total }}$ & $5.19(+0)$ \\
${ }^{16} \mathrm{O}\left(\nu, \nu^{\prime} \gamma\right){ }^{16} \mathrm{O}^{*}$ & $3.19(-3)$ \\
$\left.{ }^{16} \mathrm{O}\left(\nu, \nu^{\prime} n\right)\right)^{15} \mathrm{O}($ g.s. $)$ & $9.73(-1)$ \\
${ }^{16} \mathrm{O}\left(\nu, \nu^{\prime} p\right){ }^{15} \mathrm{~N}($ g.s. $)$ & $1.85(+0)$ \\
${ }^{16} \mathrm{O}\left(\nu, \nu^{\prime} n \gamma\right){ }^{15} \mathrm{O}^{*}$ & $3.48(-1)$ \\
${ }^{16} \mathrm{O}\left(\nu, \nu^{\prime} n n\right){ }^{14} \mathrm{O}^{*}$ & $6.11(-3)$ \\
${ }^{16} \mathrm{O}\left(\nu, \nu^{\prime} n p\right){ }^{14} \mathrm{~N}^{*}$ & $4.40(-1)$ \\
${ }^{16} \mathrm{O}\left(\nu, \nu^{\prime} p \gamma\right){ }^{15} \mathrm{~N}^{*}$ & $1.29(+0)$ \\
${ }^{16} \mathrm{O}\left(\nu, \nu^{\prime} p p\right){ }^{14} \mathrm{C}^{*}$ & $8.35(-2)$ \\
${ }^{16} \mathrm{O}\left(\nu, \nu^{\prime} p \alpha\right){ }^{11} \mathrm{~B}^{*}$ & $9.15(-2)$ \\
${ }^{16} \mathrm{O}\left(\nu, \nu^{\prime} n \alpha\right){ }^{11} \mathrm{C}^{*}$ & $3.88(-2)$ \\
\hline \hline
\end{tabular}

also Table I) which contributes about $1 \%$ to the total charged-current reaction cross section. Thus, one expects $O(10){ }^{12} \mathrm{~N}$ events in the SK detector for a supernova in the galactic center at $10 \mathrm{kpc}$ and if $T=8 \mathrm{MeV}$ for $\nu_{e}$ indeed occurs.

Finally, in Table IV we show the cross sections for the $\bar{\nu}_{e}$-induced charged-current reaction on ${ }^{16} \mathrm{O}$, again for two temperatures $T=5 \mathrm{MeV}$ and $T=8 \mathrm{MeV}$ to show the steep temperature dependence. For the antineutrino energies involved, the reactions often lead to excited states in ${ }^{16} \mathrm{~N}$ which then dominantly decay by neutron emission. We find that about $50 \%$ of the reactions produce the stable ground state of ${ }^{15} \mathrm{~N}$ as the final nuclear state. In addition, as noted above, a sizable fraction of the $\left(\bar{\nu}_{e}, e^{+}\right)$reactions at low neutrino energies lead to particle-bound states in ${ }^{16} \mathrm{~N}$, fol-

TABLE III. Partial cross sections for charged-current neutrinoinduced reactions on ${ }^{16} \mathrm{O}$. Fermi-Dirac distributions with $T$ $=4 \mathrm{MeV}$ and $T=8 \mathrm{MeV}$ and zero chemical potential have been assumed. The cross sections are given in units of $10^{-42} \mathrm{~cm}^{2}$, exponents are given in parentheses.

\begin{tabular}{lcc}
\hline \hline Neutrino reaction & $\sigma, T=4 \mathrm{MeV}$ & $\sigma, T=8 \mathrm{MeV}$ \\
\hline total & $1.91(-1)$ & $1.37(+1)$ \\
${ }^{16} \mathrm{O}\left(\nu_{e}, e^{-} p\right){ }^{15} \mathrm{O}$ (g.s.) & $1.21(-1)$ & $6.37(+0)$ \\
${ }^{16} \mathrm{O}\left(\nu_{e}, e^{-} p \gamma\right){ }^{15} \mathrm{O}^{*}$ & $4.07(-2)$ & $3.19(+0)$ \\
$\left.{ }^{16} \mathrm{O}\left(\nu_{e}, e^{-} n p\right)\right)^{14} \mathrm{O}^{*}$ & $3.92(-4)$ & $1.76(-1)$ \\
$\left.{ }^{16} \mathrm{O}\left(\nu_{e}, e^{-} p p\right)\right)^{14} \mathrm{~N}^{*}$ & $2.61(-2)$ & $3.26(+0)$ \\
${ }^{16} \mathrm{O}\left(\nu_{e}, e^{-} \alpha\right){ }^{12} \mathrm{~N}^{*}$ & $1.16(-3)$ & $1.31(-1)$ \\
${ }^{16} \mathrm{O}\left(\nu_{e}, e^{-} p \alpha\right){ }^{11} \mathrm{C}^{*}$ & $2.17(-3)$ & $5.66(-1)$ \\
${ }^{16} \mathrm{O}\left(\nu_{e}, e^{-} n \alpha\right){ }^{11} \mathrm{~N}(p){ }^{10} \mathrm{C}^{*}$ & $1.11(-6)$ & $3.28(-3)$ \\
\hline \hline
\end{tabular}


TABLE IV. Partial cross sections for charged-current antineutrino-induced reactions on ${ }^{16} \mathrm{O}$. Fermi-Dirac distributions with $T=5 \mathrm{MeV}$ and $T=8 \mathrm{MeV}$ and zero chemical potential have been assumed. The cross sections are given in units of $10^{-42} \mathrm{~cm}^{2}$, exponents are given in parentheses.

\begin{tabular}{lcc}
\hline \hline Neutrino reaction & $\sigma, T=5 \mathrm{MeV}$ & $\sigma, T=8 \mathrm{MeV}$ \\
\hline total & $1.05(+0)$ & $9.63(+0)$ \\
${ }^{16} \mathrm{O}\left(\bar{\nu}_{e}, e^{+}\right){ }^{16} \mathrm{~N}($ g.s. $)$ & $3.47(-1)$ & $2.15(+0)$ \\
${ }^{16} \mathrm{O}\left(\bar{\nu}_{e}, e^{+} n\right){ }^{15} \mathrm{~N}$ (g.s.) & $5.24(-1)$ & $4.81(+0)$ \\
${ }^{16} \mathrm{O}\left(\bar{\nu}_{e}, e^{+} n \gamma\right){ }^{15} \mathrm{~N}^{*}$ & $1.47(-1)$ & $1.90(+0)$ \\
${ }^{16} \mathrm{O}\left(\bar{\nu}_{e}, e^{+} n p\right){ }^{14} \mathrm{C}^{*}$ & $4.56(-3)$ & $1.38(-1)$ \\
${ }^{16} \mathrm{O}\left(\bar{\nu}_{e}, e^{+} n n\right){ }^{14} \mathrm{~N}^{*}$ & $5.50(-3)$ & $1.81(-1)$ \\
${ }^{16} \mathrm{O}\left(\bar{\nu}_{e}, e^{+} \alpha\right){ }^{12} \mathrm{~B}^{*}$ & $1.07(-2)$ & $1.91(-1)$ \\
${ }^{16} \mathrm{O}\left(\bar{\nu}_{e}, e^{+} n \alpha\right){ }^{11} \mathrm{~B}^{*}$ & $6.20(-3)$ & $2.16(-1)$ \\
\hline \hline
\end{tabular}

lowed then by the $\beta$ decay of the ${ }^{16} \mathrm{~N}$ ground state. For the antineutrino supernova spectrum with $T=5 \mathrm{MeV}$ we calculate a partial cross section of $3.5 \times 10^{-43} \mathrm{~cm}^{2}$. This amounts to about $5 \%$ of the reaction cross section expected from the neutral-current signal proposed in Ref. [4], considering that there are four supernova $\nu_{x}$ species which can induce this signal. In Refs. [4,25] it has been estimated that the neutralcurrent signal corresponds to about 700 events in SK for a supernova at $10 \mathrm{kpc}$, e.g., in our galactic center. Using this estimate, we expect about 40 supernova $\bar{\nu}_{e}$-induced events in $\mathrm{SK}$ leading to the ${ }^{16} \mathrm{~N}$ ground state and which can be identified by the delayed $\beta$ decay. Note, however, that this event rate corresponds to less than $1 \%$ of the total supernova neutrino rate in SK, with positrons being produced by the $\bar{\nu}_{e}$ $+p \rightarrow e^{+}+n$ reaction giving the dominating signal. Thus, it is unlikely that $\beta$ decays from ${ }^{16} \mathrm{~N}$, generated by ${ }^{16} \mathrm{O}\left(\bar{\nu}_{e}, e^{+}\right){ }^{16} \mathrm{~N}$ in the supernova $1987 \mathrm{~A}$, were observed by the Kamiokande detector. The partial ${ }^{16} \mathrm{O}\left(\bar{\nu}_{e}, e^{+}\right){ }^{16} \mathrm{~N}$ reaction cross section increases by more than a factor of 6 , if the $\bar{\nu}_{e}$ temperature is increased from 5 to $8 \mathrm{MeV}$. The observation of the ${ }^{16} \mathrm{~N} \beta$ decay would then constitute a sizable and clean signal for SK.

Another interesting reaction, leading to an observable $\beta$ decay in $\mathrm{SK}$, is ${ }^{16} \mathrm{O}\left(\bar{\nu}_{e}, e^{+} \alpha\right){ }^{12} \mathrm{~B}$ (see Table $\mathrm{I}$ ). One would expect $O(10){ }^{12} \mathrm{~B}$ decays in the SK detector for a supernova in the galactic center at $10 \mathrm{kpc}$ and if $T=8 \mathrm{MeV} \bar{\nu}_{e}$ temperature occurs.

In conclusion, our calculation of the $\nu$-induced reactions on ${ }^{16} \mathrm{O}$ confirms that, as has been suggested by Nussinov and Shrock [5], many of these reactions lead to $\beta$-unstable nuclei in the final channel. Of particular interest here are nuclei such as ${ }^{16} \mathrm{~N},{ }^{12} \mathrm{~B}$, and ${ }^{12} \mathrm{~N}$ as their $Q_{\beta}$ values are large enough for the subsequent decay to be observable in the Super-Kamiokande detector. We have calculated various reaction paths leading to these nuclei. For typical atmospheric neutrino energies our evaluation of the corresponding cross sections suggests, unfortunately, that the production of these nuclei in Super-Kamiokande is considerably less likely than Ref. [5] estimated. For supernova neutrinos, however, the ${ }^{16} \mathrm{O}\left(\bar{\nu}_{e}, e^{+}\right){ }^{16} \mathrm{~N}$ reaction can produce an observable, additional signal in Super-Kamiokande for supernovae from within our galaxy.

Discussions with R. Shrock are gratefully acknowledged. The work has been partly supported by the Danish Research Council. P.V. thanks the Institute of Physics and Astronomy at the University of Aarhus for its hospitality.
[1] Y. Fukuda et al., Phys. Rev. Lett. 81, 1562 (1998).

[2] Y. Fukuda et al., Phys. Rev. Lett. 81, 1158 (1998).

[3] K. S. Hirata et al., Phys. Rev. Lett. 58, 1490 (1987).

[4] K. Langanke, P. Vogel, and E. Kolbe, Phys. Rev. Lett. 76, 2629 (1996).

[5] S. Nussinov and R. Shrock, Phys. Rev. Lett. 86, 2223 (2001).

[6] E. Kolbe, K. Langanke, F.-K. Thielemann, and P. Vogel, Phys. Rev. C 52, 3437 (1995).

[7] K. Nakayama, S. Drozdz, S. Krewald, and J. Speth, Nucl. Phys. A470, 573 (1987).

[8] E. Kolbe, K. Langanke, S. Krewald, and F.-K. Thielemann, Nucl. Phys. A540, 599 (1992).

[9] J. J. Cowan, F. K. Thielemann, and J. W. Truran, Phys. Rep. 208, 208 (1991); T. Rauscher and F.-K. Thielemann, At. Data Nucl. Data Tables 75, 1 (2000).

[10] E. Kolbe, K. Langanke, and P. Vogel, Phys. Rev. C 50, 2576 (1994).

[11] E. Kolbe, K. Langanke, and P. Vogel, Phys. Rev. C 62, 055502 (2000).

[12] J. Engel, E. Kolbe, K. Langanke, and P. Vogel, Phys. Rev. D
48, 3048 (1993).

[13] F. R. Kane, M. Eckhause, G. H. Miller, B. L. Roberts, M. Vislay, and R. E. Welsh, Phys. Lett. 45B, 292 (1973).

[14] W. C. Haxton, Phys. Rev. D 36, 2283 (1987).

[15] M. Goldhaber, as quoted in Ref. [5].

[16] S. E. Woosley, J. R. Wilson, G. J. Mathews, R. D. Hoffman, and B. S. Meyer, Astrophys. J. 433, 229 (1994).

[17] A. Mezzacappa et al., Astrophys. J. 495, 911 (1998).

[18] S. Yamada, H. T. Janka, and H. Suzuki, Astron. Astrophys. 344, 533 (1999).

[19] A. Burrows et al., Astrophys. J. 539, 865 (2000).

[20] Q. R. Ahmad et al., Phys. Rev. Lett. 87, 071301 (2001).

[21] R. Bionta et al., Phys. Rev. Lett. 58, 1494 (1987).

[22] A. Yu. Smirnov, D. Spergel, and J. N. Bahcall, Phys. Rev. D 49, 1389 (1994).

[23] D. B. Cline, Nucl. Phys. B (Proc. Suppl.) 100, 58 (2001).

[24] C. Lunardini and A. Yu. Smirnov, Nucl. Phys. B616, 307 (2001).

[25] J. F. Beacom and P. Vogel, Phys. Rev. D 58, 053010 (1998). 